Revista de Comunicación y Salud, 2021 Vol. 11, no 1, 77-102

Editado por Cátedra de Comunicación y Salud

ISSN: 2173-1675

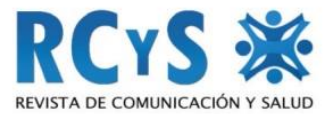

Enviado 08/03/2021

Aprobado 13/04/2021

Publicado 28/04/2021

\title{
NUEVAS NECESIDADES COMUNICATIVAS DEL PACIENTE Y SU RELACIÓN CON EL COACHING PARA PACIENTES
}

New patient communication needs and its relationship with coaching for patients

Teresa Ferreiro Vilariño ${ }^{1}$

Universidad Complutense de Madrid. España

teresa@teresaferreiro.com

\section{Cómo citar el artículo}

Ferreiro Vilariño, T. (2021). Nuevas necesidades comunicativas del paciente y su relación con el coaching para pacientes. Revista de Comunicación y Salud, 11, 77102. https://doi.org/10.35669/rcys.2021.11.e274

\section{Resumen}

A lo largo de la historia, las necesidades de los pacientes han ido evolucionando, y con ellas, los aspectos comunicativos y relacionales que definen la relación médico-paciente. Este artículo analiza los modelos relacionales de diferentes autores y sus características comunicativas, explorando las habilidades de comunicación necesarias en cada caso para, a partir de ahí, profundizar en el perfil del paciente del siglo XXI, caracterizado por su deseo de empoderarse y adquirir herramientas que le permitan tomar las riendas de su proceso y decidir con criterio. En una segunda parte, se presenta el coaching como nuevo modelo relacional. La confusión en torno al coaching de salud tanto desde el punto de vista de la metodología de trabajo como de los objetivos que persigue en su trato con el paciente deja un vacío en cuanto a la aproximación hacia el paciente. Por este motivo, y apoyándose en la investigación realizada con pacientes de cáncer de mama, se propone el coaching para pacientes como nuevo modelo relacional, un tipo de coaching con características propias y específicas que dirige su mirada hacia el futuro y que permite al individuo, por medio de herramientas prácticas, recuperar la confianza y dedicarse un espacio de autorreflexión y crecimiento.

1 Coach profesional certificada y afectada con cáncer de mama. Representante de pacientes, conferenciante y formadora. Doctoranda en la Universidad Complutense de Madrid. 
Nuevas necesidades comunicativas del paciente y su relación con el coaching para pacientes

Palabras clave: Empoderamiento; comunicación; salud; cáncer; paciente; coaching; desarrollo personal

\begin{abstract}
Throughout history, patients' needs have evolved, and with them, the communication and relational aspects that define the doctor-patient relationship. This article analyzes the relational models from different authors and their communicative characteristics, analyzing the communication skills necessary in each case to, from there, delve into the profile of the 21st century patient, characterized by their desire to feel empowered and acquire tools that allow them to take control of their process and decide wisely. As a second step, coaching is presented as a new relational model. The confusion surrounding health coaching, both from its working methodology perspective as for its objectives when dealing with the patient, leaves a void in terms of the approach to be used. This is why, and based on the research carried out with breast cancer patients, this article proposes coaching for patients as a new relationship model and a new type of coaching with its own and specific characteristics, that puts on the spotlight the patients' future possibilities and allows the individual, through practical tools, to regain confidence and dedicate a space for self-reflection and growth.
\end{abstract}

Keywords: Empowerment; communication; healt; cancer; patient; coaching; personal development

\title{
1. INTRODUCCIÓN
}

El perfil del paciente ha ido evolucionando con el paso de los años, al igual que su relación con el profesional de la salud. Hoy en día, las organizaciones relacionadas con la salud tanto dentro del ámbito público como del privado, así como el propio paciente, hacen referencia a un perfil de persona empoderada y activa, que desea tomar el control de su situación y tomar decisiones con conocimiento y criterio. Los modelos de relación médico-paciente más conocidos y citados hasta la fecha reflejan las distintas personalidades y a menudo expectativas de cada uno de los interlocutores, sin entrar a valorar el cómo crear pacientes activos. La formación y el posterior reciclaje para un desarrollo profesional (Barrientos-Báez et al., 2019) de la medicina se hace fundamental de cara a la relación médico-paciente.

La investigación realizada en este estudio pone de manifiesto que las metodologías utilizadas hasta ahora en la formación de pacientes empoderados no responden enteramente a la necesidad propia del paciente de tomar las riendas de su enfermedad y de su vida. La Academia también ha tomado cartas en el asunto y propone nuevos planes de formación para tratar situaciones médicas especiales (Carretero-Díaz y Barrientos-Báez, 2019) y más en extenso, la formación multimedia para labores sociales (López Meneses et al., 2019). 
Nuevas necesidades comunicativas del paciente y su relación con el coaching para pacientes

Este artículo presenta una revisión de los distintos modelos de relaciones y el tipo de comunicación prevaleciente en cada uno de ellos. A partir de los modelos clásicos, se profundiza en el uso de la disciplina del coaching profesional para lograr el objetivo de empoderamiento de los pacientes algo que ya existía avant la lettre en casos de motivación para públicos específicos (Odor Hurtado, 2020). Su análisis también muestra confusión y carencias en lo que respecta al coaching de salud y su proceso de empoderamiento del paciente.

Por ello, se expone como conclusión de este artículo el reciente trabajo llevado a cabo por Ferreiro (2021) con pacientes de cáncer de mama, que presenta un nuevo modelo de relación con el paciente (Cofré Soto, 2020), el coaching para pacientes, centrado en dotar a estas personas de herramientas para empoderarse y autorizarse a sí mismas para tomar decisiones, a través de un proceso de trabajo personal y la búsqueda de respuestas llevado a cabo en colaboración con un profesional del coaching, más que a través de un proceso educativo realizado por profesionales de la salud. Esta necesidad de cambio de paradigma en la comunicación médico-paciente es ya una constante en muchos de los ámbitos de la salud, como es el caso de la gestión de enfermedades graves (Cofré Soto, 2020), crónicas (Compte-Pujol et al., 2020), psicológicas (Vargas Delgado, 2020) o discapacidades (Lucas Moreno, 2020). Dejamos en este punto un frente abierto para futuras investigaciones pero de crucial interés: la gestión de la inteligencia emocional (IE). Un término que Barrientos-Báez (2019a) define como la capacidad de controlar y gestionar positivamente las emociones propias y ajenas, en un escenario cualquiera, donde se producen experiencias y cambios como parte del proceso de aprendizaje personal. De aquí se desprende la importancia de la IE en los profesionales de enfermería por competencias y ámbito geográfico (Veliz et al., 2018).

\section{OBJETIVOS Y METODOLOGÍA}

A partir de la revisión de los tipos de relación existentes entre el médico y el paciente, el presente estudio se plantea como objetivo inicial analizar las necesidades comunicativas y relacionales del paciente del siglo XXI, eminentemente aquellas que le ayudan en su proceso de empoderamiento.

En base a este análisis, un segundo objetivo del artículo es el de profundizar en las habilidades de comunicación necesarias en la relación con el paciente, incluyendo entre ellas algunas que sí bien no son estrictamente comunicativas sino derivadas del coaching profesional, sí contribuyen a mejorar relación con el paciente y su proceso de empoderamiento.

El objetivo último de este estudio consiste en explorar y fundamentar el uso de la disciplina del coaching profesional en la relación entre médico y paciente, proponiendo el coaching para pacientes como nuevo modelo de relación entre ambos. La revisión del coaching, y más concretamente, del coaching de salud, revela una serie de carencias a la hora de hacer frente a las nuevas necesidades comunicativas del paciente. Por ello se propone el coaching para pacientes, un modelo con características específicas 
Nuevas necesidades comunicativas del paciente y su relación con el coaching para pacientes

destinadas a acompañar al paciente y dotarle de herramientas en su camino hacia el empoderamiento.

Se ha empleado como metodología la revisión bibliográfica de fuentes especializadas, provenientes tanto del entorno de la salud como del de la comunicación. El punto de partida del proceso de documentación ha sido el artículo de Emanuel y Emanuel (1999, pp. 109-126) que establece cuatro modelos relacionales para, en base a su clasificación, profundizar en su evolución y conocer las nuevas propuestas de autores posteriores. En cuanto a las fuentes relacionadas con el coaching, al ser esta disciplina muy reciente (cuenta con unos 30 años de existencia), ha sido menos tratada por la literatura académica, por lo que se ha recurrido a bibliografía de coaches prestigiosos y publicaciones en línea.

\section{EL PACIENTE $Y$ LOS DISTINTOS MODELOS RELACIONALES $Y$ DE COMUNICACIÓN}

\subsection{Distintos modelos de relación médico paciente}

Existen numerosos modelos de relación médico-paciente que han convivido a lo largo de los años. Si bien algunos de ellos pueden darse más frecuentemente en determinados períodos históricos, su existencia no viene necesariamente determinada por el momento histórico. En su obra Las Leyes (pp. 720-857), Platón ya establecía diferencias entre la relación médico-paciente cuando éste último era un esclavo o cuando era un hombre libre.

En línea con el desarrollo de los diferentes modelos -tecnológicos las más de las veces (Rando Cueto et al., 2021; Sancho Escrivá et al., 2020)- que actualmente conviven en diferentes entornos médicos -incluyendo los hospitalarios (Barquero Cabrero et al., 2018)-, la forma en que los pacientes se han posicionado frente a su salud ha evolucionado desde un posicionamiento pasivo a otro en el que el paciente adquiere responsabilidad y compromiso frente a sus decisiones (Barrientos-Báez, 2019b) aunque las fuentes de información no sean veraces, como el caso de las campañas antivacunas (Piqueiras Conlledo et al., 2020). Es el caso de los pacientes empoderados, o competentes, aunque con fuentes informativas a veces dudosas que generan estereotipos, lo que dificulta la labor de los profesionales ante los pacientes (Lara Martínez y Lara Martínez, 2019) al verse superados por quienes manejan las Redes, como ha sido el caso de la reciente pandemia durante su primera época (García-Manso, 2021; López del Castillo Wilderbeek, 2021).

Emanuel y Emanuel (1999, pp. 109-126) describen al menos cuatro modelos de relación médico-paciente:

- Modelo paternalista: Una vez establecido el diagnóstico, el médico determina qué es lo mejor para el paciente y para su salud. Le da información seleccionada de los tratamientos que considera idóneos para su salud, dirigiéndole hacia donde, según su criterio, debe ir. Este médico, que actúa como tutor del paciente, tiene también obligaciones, como por ejemplo poner los intereses del paciente por

Revista de Comunicación y Salud, 2021, Vol. 11, 77-104 
Nuevas necesidades comunicativas del paciente y su relación con el coaching para pacientes

encima de los propios. "Se concibe la autonomía del paciente como un asentimiento (...) a lo que el médico considera que es mejor" (Emanuel y Emanuel, 1999).

- Modelo informativo: Es un modelo técnico, caracterizado por el empleo de una gran cantidad de información científica. El médico proporciona al paciente datos relativos a su enfermedad, diferentes tratamientos, con los riesgos y beneficios que cada una conlleva. En este modelo los valores del paciente están presentes, al margen de la relación con su médico. Se concibe la autonomía del paciente "como el control del mismo sobre la toma de decisiones médicas" (Emanuel y Emanuel, 1999).

- Modelo interpretativo: En este caso, "el objetivo de la relación médico-paciente es determinar los valores del paciente y qué es lo que realmente desea (...) y ayudarle así a elegir de entre todas las intervenciones médicas disponibles aquellas que satisfagan sus valores" (Emanuel y Emanuel, 1999). Es decir, que los valores del paciente pueden estar poco definidos o entrar en conflicto con su experiencia, y es papel del médico ayudarle a comprenderlos, como consejero, sin juzgar. "El paciente llega a conocer más claramente quién es y cómo las distintas opciones médicas afectan a su identidad" (Emanuel y Emanuel, 1999).

- Modelo deliberativo: En este modelo, la relación-médico paciente ayuda al paciente a elegir, de entre sus valores relacionados con la salud, aquellos que son más importantes a tener en cuenta en el momento en que se encuentra. El médico actúa de maestro o amigo, indicando lo que el paciente podría hacer y lo que cree que encaja mejor con su persona, pero dejando en manos del paciente la decisión final. "La autonomía del paciente se concibe como un autodesarrollo moral. El paciente está capacitado, no solamente para seguir unas preferencias irreflexivas (...) sino también para analizar, a través del diálogo, los distintos valores relacionados con la salud, su importancia, y sus implicaciones en el tratamiento" (Emanuel y Emanuel, 1999).

Estos cuatro modelos conviven en espacio y tiempo. Un mismo médico puede optar por uno u otro en función de la situación. Por ejemplo, el modelo paternalista es el más utilizado en casos de extrema urgencia, donde hace falta tomar decisiones importantes en muy poco tiempo.

Al ir avanzando en cada uno de estos cuatro modelos, se aprecia una evolución en los roles de médico y paciente. De un modelo paternalista inicial, rígido en que la relación entre el médico y el paciente es asimétrica y priva a este último de su autonomía y su capacidad de elección, se va pasando a modelos más igualitarios, en que entran en juego conceptos como conceptos como autonomía, libertad, respeto, confianza y confidencialidad. Desde mediados del siglo XX el médico adquiere una dimensión más humana, sin dejar por ello de lado su capacidad técnica, y el paciente gana en poder y reconocimiento ante los ojos del profesional. El paciente se convierte además en una pieza clave durante su enfermedad y en el proceso de curación.

Un médico debe poseer no solamente el conocimiento y habilidades técnicas, sino también una comprensión de la naturaleza humana. El paciente no es sólo un grupo de síntomas, órganos dañados y emociones alteradas. El paciente es un ser

Revista de Comunicación y Salud, 2021, Vol. 11, 77-104 
Nuevas necesidades comunicativas del paciente y su relación con el coaching para pacientes

humano, al mismo tiempo preocupado y esperanzado, que busca alivio, ayuda y confianza. La importancia de una relación íntima entre el paciente y su cuidador no puede nunca ser calificada como exagerada, porque en la mayoría de los casos, tanto un diagnóstico adecuado como su tratamiento efectivo dependen de la calidad de esta relación (Hellín, 2002, pp. 450-454).

Además de la clasificación de los modelos de Emanuel, los autores Kabaa y Sooriakumaran (2007, pp. 57-65) recogen los tres modelos básicos de relación médico paciente presentados por Szasz y Hollender (1956, pp. 585-592):

- Actividad - pasividad: Se percibe al paciente como incapaz y necesitado del conocimiento experto del doctor. El tratamiento se ejecuta al margen de la contribución del paciente y de su resultado. Esta aproximación está justificada en casos agudos.

- Cooperación de orientación: Consciente de su sufrimiento y buscando alivio, el paciente puede y quiere participar, colocando al médico en una posición de poder en que el paciente obedece sin cuestionamientos. Se da en casos de enfermedad no tan agudos.

- Participación mutua: Se basa en la idea de la igualdad entre seres humanos. En este modelo el médico puede admitir que no sabe exactamente qué es lo mejor para el paciente. La interacción entre ambos se basa en la igualdad de poder, independencia mutua y búsqueda común de la satisfacción, mediante una relación de colaboración empática y cercana a la amistad. Es el modelo ideal en enfermedades crónicas.

Tanto los modelo interpretativo y deliberativo de Emanuel como el de participación mutua de Szasz y Hollender, marcan el camino hacia un modelo centrado en el paciente (Mead y Bower, 2000, pp. 1087-1110). A partir del análisis de la literatura conceptual y empírica, Mead y Bower (2000) identifican cinco dimensiones en este nuevo modelo (Kabaa y Sooriakumaran, 2007):

- Perspectiva biopsicosocial: Se refiere al deseo de involucrarse en las dificultades que los pacientes traen a la consulta, y no sólo de sus problemas biomédicos.

- El paciente como persona: Para entender la enfermedad y aliviar el sufrimiento del paciente, los médicos necesitan entender primero el significado de esa enfermedad para ese paciente en concreto.

- Poder y responsabilidad compartida: Una relación de iguales en que el paciente tiene voz y se involucra en su tratamiento, animado por el médico.

- Alianza terapéutica: Se da gran importancia a la relación personal entre el médico y el paciente, que puede aumentar entre otras la adherencia al tratamiento y ser fundamental en el proceso de mejora y curación (Pinto et al., 2012, pp. 77-87).

- El médico como persona: El paciente y el médico se influyen mutuamente, dando espacio a las emociones del profesional como parte de los aspectos relacionales entre ambos. 
El modelo centrado en el paciente existe desde hace aproximadamente 30 años y supone objeto de estudio de un sinfín de trabajos académicos. Este modelo, que pone al paciente en el centro y determina los temas de importancia para ellos a través de la consulta y diálogo directo (Yeoman et al., 2017, pp. 76-83), marca la tendencia de los organismos institucionales y sociales, que centran sus investigaciones en cómo lograr implantar este modelo en un entorno cada vez más caracterizado por el impacto de la tecnología por un lado (incluyendo aspectos como la diseminación de información a través de internet, las nuevas máquinas cada vez más sofisticadas, el impacto de la inteligencia artificial, la disrupción digital, etc.), y por una búsqueda de rentabilidad del sistema que trae consigo la reducción del tiempo que el médico puede dedicar a cada paciente, que dificulta el poder construir una relación humana entre ambos actores por lo que es muy recomendable trabajar la inteligencia espiritual como recurso que optimice ese tiempo (Alarcón-Orozco, 2020).

\subsection{Evolución histórica del paciente y sus tipologías}

Históricamente, el paciente ha sido un sujeto pasivo que se avenía al modelo paternalista. Acudía a la consulta del médico para escuchar sus recomendaciones respecto a lo que tenía que hacer para curarse, sin implicarse en el proceso de curación. En el siglo XIX, con la llegada de la psicología y a partir de las teorías de Freud, comienza a percibirse al paciente como una persona. Por tanto, es fundamental escucharle cuidadosamente, creando una relación de comunicación sincera y reintroduciendo al paciente en la consulta como participante activo (Kabaa y Sooriakumaran, 2007).

Cuando a mediados del siglo XX cobran peso conceptos eminentemente humanistas como autonomía, libertad, respeto, confianza y confidencialidad, las fuerzas de médico y paciente tienden a igualarse. El año 1973 es clave en esta evolución histórica del paciente, pues es entonces cuando la Asociación Americana de Hospitales aprueba la primera Carta de Derechos del Paciente, que supone el reconocimiento oficial del derecho del enfermo a recibir una completa información sobre su situación clínica y a decidir entre las opciones posibles, como adulto autónomo y libre que es (Lázaro y Gracia, 2006, pp. 7-17). Desde entonces, la figura del paciente ha ido evolucionando y afianzándose, hasta convertirse en un paciente más comprometido con su salud, más responsable y activo. 
Nuevas necesidades comunicativas del paciente y su relación con el coaching para pacientes

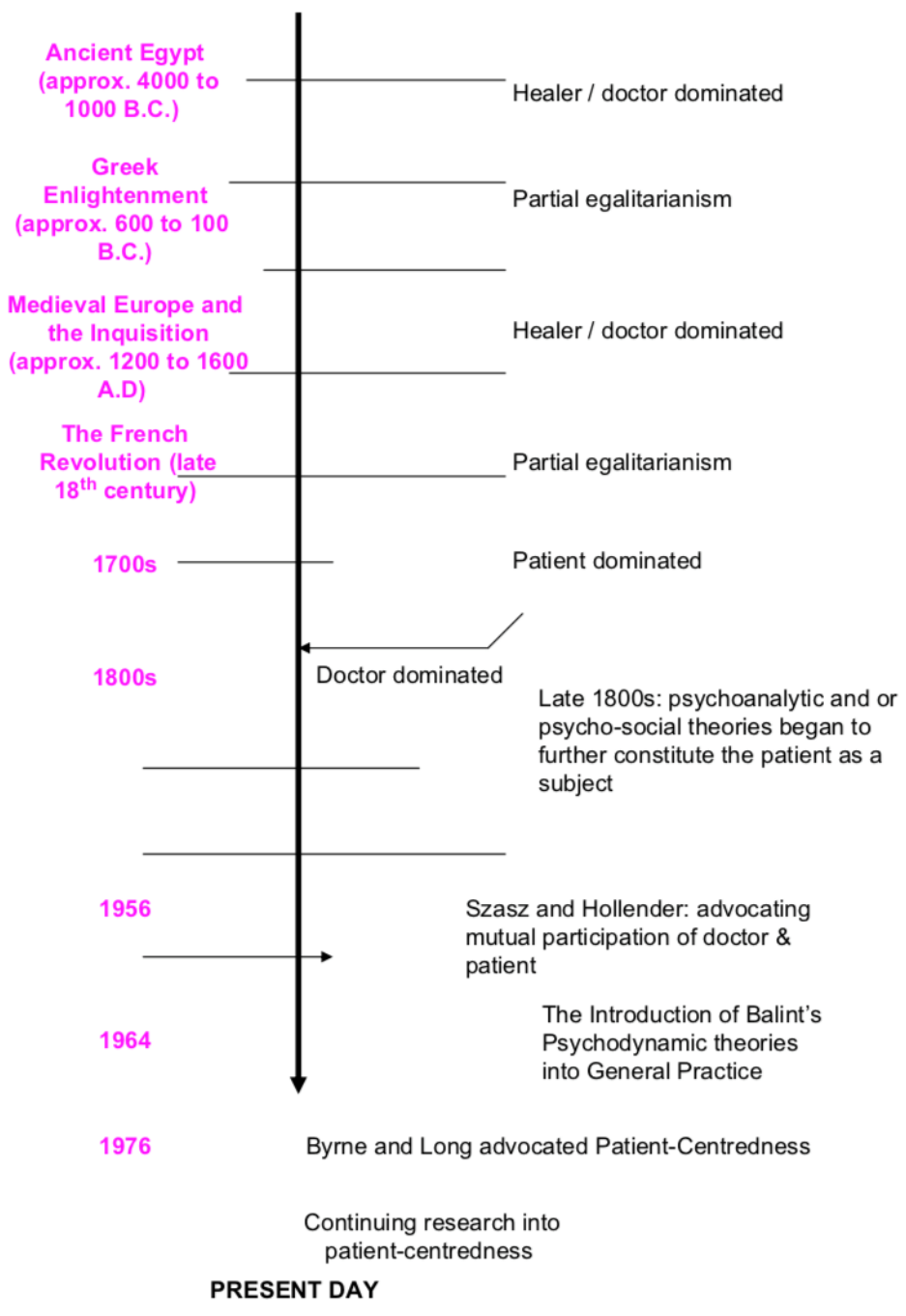

Gráfico 1: Evolución de la figura del paciente Fuente: Kabaa y Sooriakumaran, 2007

El desarrollo de Internet ha introducido grandes cambios en la relación entre médico y paciente. Ferguson acuñó en 2017 el término e-patient para describir individuos equipados, empoderados, capacitados y comprometidos, que utilizan Internet para buscar información acerca de un tema de salud tanto para ellos como para alguien de su entorno (Ferguson y e-Patients Scholars Working Group, 2007). Con la información que obtienen a través de Internet, los e-patients tienen la oportunidad de entender mejor su enfermedad y hacer preguntas relevantes a sus médicos. Además, entienden que sus médicos no pueden estar al corriente de todos los avances y ofrecen ideas y soluciones. Hoy en día el término ha caído en desuso y la definición se corresponde con la del paciente activo. 
Nuevas necesidades comunicativas del paciente y su relación con el coaching para pacientes

\subsection{El paciente del siglo XXI}

Esta evolución en la actitud y las necesidades del paciente trae consigo como consecuencia la necesidad de que la relación entre el médico y paciente también cambie. El paciente del siglo XXI no busca en los médicos a una persona con actitud paternalista, sino a "compañeros de viaje, consejeros que les ayuden a interpretar sus propios deseos, necesidades $y$ valores, y que les ayuden a decidir libre e informadamente" (Emanuel y Emanuel, 1999).

En su análisis de la evolución del tipo de paciente, Simón cita a Jadad et al. (2006) para explicar que "el papel clave del profesional sanitario del futuro no será el de procurar información científica, sino el de dar consejo y apoyo en el proceso de toma de decisiones clínicas" (Simón, 2006, pp. 29-40).

Desde comienzos del siglo XXI existe la figura del paciente experto, aquel que "transmite conocimientos y comparte experiencias con otras personas que sufren el mismo problema de salud" (Programa Pacient Expert Catalunya, 2013). Es "aquella persona afectada por una enfermedad crónica que es capaz de responsabilizarse y cuidarse, sabiendo identificar los síntomas, responder a ellos apropiadamente y adquiriendo las habilidades que le ayuden a gestionar el impacto físico, emocional y social de la patología mejorando así su calidad de vida (Mestre, 2015)".

\subsection{La comunicación en los diferentes modelos relacionales}

Los cuatro modelos de relación médico-paciente vienen acompañados por una evolución en la forma en que se comunican ambos protagonistas de esta relación.

En el modelo paternalista, la comunicación es prácticamente unidireccional. El médico experto habla y el paciente se limita a escuchar y ejecutar los mandatos el experto. La información que proporciona el médico al paciente está sesgada, ya que su objetivo es que acepte la decisión del doctor (Kabaa y Sooriakumaran, 2007). El paciente otorga al médico el poder. En su rol de tutor, el profesional puede apelar a recursos como son el tono condescendiente, la calidez o la firmeza.

El modelo informativo se caracteriza por una comunicación centrada en los hechos. Las emociones se neutralizan y el médico se posiciona como un canal transmisor de información veraz, dejando al paciente el poder de decisión final.

El tipo de comunicación empleado en el modelo interpretativo es más filosófico. Por medio de preguntas y de la selección de la información que considera más adecuada para el paciente, el médico acompaña al paciente en su búsqueda de una respuesta, por medio de preguntas y consejos. El tono es cálido y cómplice.

En el modelo deliberativo, el médico se sirve de la comunicación para transformar su rol en el de un amigo que a la vez es maestro. Ambos actores están al mismo nivel en la búsqueda de un objetivo final compartido por ambos. 
Nuevas necesidades comunicativas del paciente y su relación con el coaching para pacientes

Hoy en día se habla cada vez más del médico-coach. El término se inspira en el entorno empresarial, en que el líder-coach define un estilo de liderazgo caracterizado por la capacidad de inspirar al equipo y de potenciar las habilidades de sus miembros. Así, el médico-coach es un profesional dialogante y empático, que confía en los recursos de su paciente y le ayuda a maximizar sus posibilidades, en un clima de confianza que permite al paciente ser consciente de sus capacidades y creer en ellas. La comunicación del médico-coach está abierta al respeto. Es bidireccional y abierta, y se establece en una situación entre iguales en que ambos se sienten con capacidad de expresar sus necesidades y opiniones.

\subsection{La comunicación, elemento clave en la relación médico-paciente}

Una buena relación y una buena comunicación entre el médico y el paciente tienen resultados muy positivos en la evolución de la enfermedad. Un estudio realizado en 2002 entre 110 pacientes demostró que una comunicación centrada en el paciente mejoraba ostensiblemente el estado de salud del paciente tanto sus niveles de ansiedad como la intensidad del dolor (Muñoz et al., 2002, pp. 23-31). Autores más recientes afirman con rotundidad que "la efectividad de un tratamiento médico depende de la calidad de la relación médico-paciente" (McCabe y Healey, 2018, pp. 409-424). Esta relación se basa en la confianza (Pellegrini, 2017, pp. 95-102), y la confianza se construye gracias a una buena comunicación entre los interlocutores propiciada por un conjunto de habilidades que los profesionales pueden adquirir (Honavar, 2018, pp. 1527-1528).

Asnani, añade que "las investigaciones han demostrado abundantemente que no importa cuán profundos sean los conocimientos del médico, si no puede abrir un buen canal de comunicación con el paciente, no podrá ser de ayuda alguna a este último" (Asnani, 2009, pp. 357-361). Sin embargo, los médicos pueden no ser conscientes de cómo viven los pacientes su estilo de comunicación (Burt et al., 2018, pp.330-337).

Por otra parte, es comúnmente aceptado el hecho de que muchos médicos tienen dificultades para comunicarse con sus pacientes (Asnani, 2009). Resulta especialmente interesante ver cómo las habilidades de comunicación de los médicos descienden a medida que progresa su formación en la facultad de medicina (DiMatteo, 1998). Además, la dureza física y emocional de la formación, sobre todo durante la especialidad y la residencia, elimina la empatía y reemplaza la conversación por el empleo de técnicas y procedimientos (DiMatteo, 1998).

\subsection{Habilidades de comunicación efectivas en la relación con el paciente}

Entre las habilidades de comunicación que se consideran más importantes en la literatura académica para mantener una buena comunicación con el paciente, destacan la escucha activa, el uso de preguntas abiertas, y la capacidad para empatizar (Fong Ha y Longnecker, 2010), con el fin de crear una comunicación colaborativa que asegura el intercambio real de información. Para ello, el médico también necesitará ser capaz de facilitar una discusión honesta en que el paciente se sienta escuchado, recurriendo para

Revista de Comunicación y Salud, 2021, Vol. 11, 77-104 
Nuevas necesidades comunicativas del paciente y su relación con el coaching para pacientes

ello a menudo a habilidades de negociación. Al poner en práctica estas habilidades, el médico centra su comunicación en el paciente, participando así de un modelo de relación médico-paciente centrado en el paciente. La mirada, juega también un importante papel tanto a la hora de comunicar como a la hora de crear el contexto que permite que afloren las emociones de los pacientes.

\section{- Escucha activa}

Uno de los pilares fundamentales de la comunicación es la escucha. Escuchar de forma activa se refiere a la capacidad para escuchar permitiendo que la otra persona se exprese en su totalidad. La escucha activa presta atención a las palabras, a los gestos y a las emociones. Cuando los médicos la practican, satisfacen la necesidad de los pacientes de sentir que se les conoce y son comprendidos (Karora, 2003, pp. 38-43), escuchados y valorados y la evolución de su enfermedad es más positiva (DiMatteo, 1998).

Desde el punto de vista del coaching, una buena escucha facilita la apertura de las personas (Kimsey-House et al., 2011), porque perciben el interés del otro, y su presencia. La escucha ayuda a construir un entorno de confianza y seguridad, en el que las personas se sienten respetadas y valoradas.

La escucha no es un acto pasivo, sino activo. La escucha activa es aquella en que el receptor está presente y disponible para la otra persona, implicándose en el proceso y utilizando herramientas para en muchos casos ayudar al emisor a conectar con aquello que es importante, eliminando las capas de "ruido" que interfieren.

\section{- Empatía}

Si bien no es una habilidad de comunicación en sí misma, es una de las habilidades clave que marcará el desarrollo de la relación médico-paciente. La empatía es la capacidad para ponerse en lugar de la otra persona y comprender lo que está sintiendo. En el caso de la relación entre médico y paciente, cuando el médico reconoce que entiende la perspectiva del paciente, el paciente se siente más escuchado y acompañado.

Cuando Goleman definió las habilidades interpersonales (Goleman, 1996), diferenció la empatía (habilidad emocional) de otras habilidades relacionales basadas en la comunicación. Partiendo de esta definición, una persona empática se sirve de la comunicación no verbal para transmitir esta empatía. Es por ello que incluimos la empatía como una de las habilidades necesarias para una relación exitosa.

A grandes rasgos, la empatía consiste en percibir lo que sienten y piensan los demás sin que nos lo digan. O en ser capaces de entender el sentimiento del otro y ponernos en su lugar. Se habla de comunicación empática cuando alguna de las cualidades de la empatía pone al servicio de la comunicación con el objetivo de abrir nuevos caminos e intercambiar sentimientos, actitudes y emociones, sin juicio. Marshall Rosenberg, 
Nuevas necesidades comunicativas del paciente y su relación con el coaching para pacientes

conocido pacifista, utilizó el término en 1998, en un artículo que abordaba aspectos de comunicación en el ámbito de la salud (Rosenberg y Molho, 1998, pp. 335-340). Más adelante ha seguido publicando libros en los que habla de comunicación no violenta y empatía (Rosenberg y Chopra, 2015). Otros autores también hacen referencia a la comunicación empática en libros y artículos (Servellen, 2009; Belles, 2018, pp. 10-11). Al describir su modelo emotivo-racional de la entrevista clínica, Borrell (2004) habla de una serie de cualidades de gran importancia para el profesional de la salud (calidez, respeto, cordialidad y empatía entre otras), a la hora de acoger al paciente y sentar las bases de la relación con él.

\section{- Preguntas abiertas}

En oposición a las preguntas cerradas, que incluyen una serie de opciones de respuesta (por ejemplo: "te gusta el cine? Si/no") y no facilitan el desarrollo del diálogo, las preguntas abiertas se responden de forma elaborada, con frases que facilitan el acceso a argumentaciones, elaboraciones, opiniones de más amplio desarrollo. Utilizándolas con los pacientes, se les obliga a verbalizar (Borrell, 2004). De esta manera, estas preguntas permiten acceder a la persona, a su historia y a sus preocupaciones, para a partir de ahí establecer una relación de diálogo y confianza basada en un objetivo común. Las preguntas abiertas se manifiestan como uno de los elementos que ayudan al paciente a manifestar sus preocupaciones (Maguire, 1999, pp. 1415-1422). Algunos autores (Lichstein, 1990, pp. 29-36) proponen utilizar estas preguntas y dejar que el paciente hable antes de comenzar con preguntas detalladas.

\section{- La mirada}

La mirada es un elemento fundamental de la comunicación no verbal. En su libro, La comunicación no verbal, Knapp (1980) construye sobre las cuatro funciones de la mirada para presentar las siguientes funciones:

- Regulación de la corriente de comunicación: Es decir, la mirada abre y cierra el canal de comunicación.

- Retroalimentación por control de las reacciones del interlocutor: Nos permite obtener información acerca de cómo está recibiendo la información nuestro interlocutor.

- Expresión de emociones: La mirada proporciona mucha información acerca de las emociones que se están expresando.

- Comunicación de la naturaleza de la relación interpersonal: Nos habla del tipo de relación que existe entre los interlocutores. Según Knapp, en estrecha relación con el mirar a las personas, se encuentra el factor de actitud positiva o negativa. Parece que miramos más a las personas que nos caen simpáticas.

Construyendo sobre la cuarta función de la mirada, ésta contribuye a crear un entorno de intimidad, que facilite la comunicación. Ver a la otra persona como es, dedicándole tiempo y permitiéndole expresarse y fallar, ayuda a crear ese espacio de acompañamiento y seguridad que anima a la persona a explorar y arriesgarse, confiando. Nos referimos a la mirada atenta. Una mirada, tal y como define Esquirol (2006), que 
Nuevas necesidades comunicativas del paciente y su relación con el coaching para pacientes

nace desde el respeto: "El movimiento de la atención no es sólo para rescatar al otro o a lo otro, sino también a uno mismo (...) la atención se convierte en la tarea del que debe empezar de nuevo, del que se sabe sujeto de responsabilidad y llamado a ser uno mismo". Aprender a mirar significa aprender a prestar atención, dirigir el foco y evitar la dispersión. La atención nos conecta con el mundo. (Esquirol, 2021)

\subsection{Paciente activo - paciente empoderado}

La Organización Mundial de la Salud define "empoderamiento del paciente" como "un proceso en el que los pacientes entienden su rol, y en el que los profesionales de la salud les dotan con el conocimiento y las habilidades para llevar a cabo su tarea, en un entorno que reconoce las diferencias culturales y comunitarias, y que fomenta la participación del paciente" (OMS, 2009).

Un paciente empoderado es aquel que es capaz de asumir su situación, tomar decisiones, resolver problemas y tomar las riendas de su vida. Así, empoderar a los pacientes implica no sólo educarlos en su enfermedad proporcionándoles la información y el conocimiento que necesitan para tan autónomos como sea posible, sino también dotarlos con los recursos necesarios para que se sientan emocionalmente capaces de tomar sus propias decisiones y fiarse de su propio criterio (Huffman, 2009, pp. 490-498). Autores como Timmermans se refiere a este tipo de paciente como "paciente comprometido", y relaciona su actitud activa al auge de Internet como fuente alternativa de información (Timmermans, 2020, pp. 259-273).

Gran parte de la literatura que profundiza en el empoderamiento del paciente y las necesidades de los enfermos lo hace desde el campo de la medicina y ciencias de la salud, no tanto desde las disciplinas de la comunicación y/o del coaching. Desde el punto de vista del coaching, el empoderamiento adquiere una dimensión más amplia, porque además de tener en cuenta la relación de la persona con la enfermedad, la capacita para gestionar sus emociones y controlar su vida de tal manera que pueda convertirla en aquello que desea vivir. Entre estas emociones se encuentra la relación del paciente con su enfermedad y el lugar que quiere que ocupe. Diversos estudios que han evaluado el impacto del empoderamiento en la satisfacción del paciente o su adherencia al tratamiento sugieren que una participación activa del paciente se asocia con una mejor recuperación (Roberts, 1999, pp. 82-92). Por otra parte, recuperar el control de sus vidas se convierte para los pacientes en uno de los pilares de su recuperación (Gray et al., 1991, pp. 33-45).

Otros ámbitos como el deportivo, demuestran la utilidad del coaching para la gestión de emociones (Correia et al., 2016; Davis y Davis, 2016, pp. 285-306), si bien en este caso la figura del coach se orienta hacia el entrenador personal y su capacidad para ayudar al deportista a gestionar sus emociones, o al profesional (Cox y Patrick, 2012, pp. 34-51).

Para que la relación médico-paciente se de a su más alto nivel, el paciente necesita conocer y practicar una serie de habilidades que se describen a continuación:

Revista de Comunicación y Salud, 2021, Vol. 11, 77-104 
Nuevas necesidades comunicativas del paciente y su relación con el coaching para pacientes

Activo: Un paciente activo toma la iniciativa, pregunta, busca y tiene una actitud abierta hacia su situación.

Curioso: Desea conocer y comprender su enfermedad para así poder hacerse cargo de la situación. Para ello, está abierto a investigar, aprender de nuevas fuentes de información al margen de su médico, lee, escucha y hace preguntas relacionadas con su enfermedad que le ayudan a adquirir ese conocimiento.

Responsable: El paciente activo sabe que la evolución de su salud depende en gran parte de su compromiso hacia su propia curación. Habrá momentos en que necesitará tomar decisiones o llevar a cabo acciones o comportamientos que pueden determinar en gran medida la evolución de su enfermedad. Utilizar la capacidad de decidir de una forma consciente implica conocer las posibles consecuencias de sus decisiones y aceptar su responsabilidad frente a ellas. De un rol en que el paciente se posiciona como víctima pasa a ocupar un rol en que se convierte en el director de orquesta.

Consciente: El paciente conoce su situación real y sabe la repercusión de los tratamientos o de su actitud. Además, sabe y acepta, las consecuencias de las decisiones que toma, llegando incluso a optar por un camino diferente al propuesto por el médico, que se adapta mejor a su persona, sus valores y creencias.

Comprometido: El paciente activo se compromete a seguir un tratamiento, un estilo de vida, unas indicaciones que le ayudan en su enfermedad. Este compromiso suele ser consigo mismo y/o con otras personas (médico, familiares, etc.)

Autónomo: Toma decisiones por sí mismo, en función de su estado, de sus necesidades, de su entorno. Sabe lo que le conviene y tiene margen de actuación para actuar sobre su enfermedad sin necesidad de consultar al médico cualquier pequeño aspecto.

Informado: Conoce su enfermedad, los tratamientos que está siguiendo y el porqué. En ocasiones está más actualizado que el propio médico (Ferguson y e-Patients Scholars Working Group, 2007).

Criterio: Las decisiones que toma el paciente activo, las opciones que elige, los comportamientos que adopta, nacen de su discernimiento. Cada persona tiene su criterio propio, que merece ser respetado. Se basa en sus valores y sus experiencias. Frente a las discrepancias que puedan existir entre médico y paciente, el rol del médico consiste en aceptar el criterio del paciente activo y acompañarlo en su decisión. 
Nuevas necesidades comunicativas del paciente y su relación con el coaching para pacientes

\section{EL COACHING COMO MODELO DE COMUNICACIÓN Y EMPODERAMIENTO DE LOS PACIENTES}

La International Coach Federation (ICF) ${ }^{2}$ define el coaching profesional como "una asociación con clientes en un proceso de acompañamiento reflexivo y creativo que les inspira a maximizar su potencial personal y profesional" (ICF, 2018).

- Una asociación con clientes: Es decir, que el coach trabaja con clientes (en oposición a los psicólogos, por ejemplo, que trabajan con pacientes). Esta relación es de igual a igual. Para que el coaching funcione es fundamental que todos los participantes sean protagonistas del proceso. En ningún caso el coach se posiciona como conocedor o sabedor de las soluciones a los problemas 0 situaciones que se ponen encima de la mesa por encima de la persona con la que trabaja.

- En un proceso de acompañamiento reflexivo y creativo: El coach ayuda a la persona, por medio de preguntas, conversaciones, reflexiones y herramientas variadas a que ella misma encuentre sus respuestas.

- Inspira a maximizar su potencial personal y profesional: Uno de los principios del coaching es que todas personas tienen capacidad para elegir cómo quieren vivir su vida, y extraer todo el jugo tanto de sí mismas como de sus experiencias. Así, con el trabajo de coaching los clientes se ven capacitados para ir más allá de las posibilidades que ellos mismos se habían impuesto, en muchos casos limitantes. Esta nueva perspectiva que adquieren de sí mismos es inspiradora porque les abre nuevas maneras de posicionarse ante la vida y de vivir sus experiencias.

Leonard, considerado como uno de los padres del coaching creía que todo el mundo era un coach (2001). En su opinión todos llevamos un mentor en nuestro interior que necesita formarse en herramientas de coaching, que definía como capacidades avanzadas de comunicación (Brock, 2017). A este concepto, Whitmore añade además que el coaching se enfoca en futuras posibilidades, no en errores pasados" (2010).

\subsection{Las diferentes acepciones del coaching de salud}

Un análisis del coaching aplicado a la salud y a los pacientes pone de manifiesto la existencia de cierta confusión, tanto en lo relativo a su forma de trabajar, como en lo relativo a los objetivos que se persiguen y a los profesionales que lo practican.

En lo que respecta a la relación que se establece con los pacientes, la literatura describe a menudo un tipo de actuación de carácter directivo, en que el profesional ejerce de guía o mentor del cliente. En el campo de la medicina esta figura se hace aún más

\footnotetext{
${ }^{2}$ ICF: organismo internacional regulador del coaching y de los profesionales que lo practican. Su objetivo principal es velar por el desarrollo de esta disciplina de forma rigurosa. ICF es la organización responsable de homologar los títulos formativos de coach profesional. También ha diseñado un código ético que proporciona el marco de referencia y las normas de conducta vinculantes para los miembros de ICF y los poseedores de credenciales.
} 
Nuevas necesidades comunicativas del paciente y su relación con el coaching para pacientes

patente. Múltiples artículos se refieren al coach de salud como aquella persona que informa y lleva de la mano al cliente a lo largo de su tratamiento en pos de una curación. Los objetivos de este coaching pueden ser semejantes a los del coaching que reconocemos en este trabajo, si bien el método difiere (Clark y Douglas, 2014, pp. 15371544). Un claro ejemplo de esta metodología en que el coach guía al paciente es el cáncer coaching tal y como se entiende en América. En este caso, el coach informa al paciente acerca de lo que puede esperar con los tratamientos, da pautas de alimentación, ejercicio físico y mental, efectos secundarios, pero no ofrece el acompañamiento profesional de un coach certificado, pues no ha recibido la formación adecuada (CBC, 2018).

Asimismo, la terminología empleada para referirse este tipo de coaching puede resultar también confusa. Se habla de coaching de salud, coaching de bienestar, coaching de salud y bienestar, sin que estas diferentes expresiones ofrezcan claras diferencias de significado (Clark y Douglas, 2014).

Bennet define el coaching de salud como aquel proceso que se utiliza para "ayudar a los pacientes a obtener los conocimientos, herramientas y confianza para convertirse en participantes activos de su cuidado y puedan alcanzar los objetivos de salud fijados por ellos mismos" (Bennet et al., 2010, pp. 24-29).

Molins, médico de profesión, define el coaching de salud como aquel que "facilita que la persona consiga sus objetivos de salud de una manera más eficaz. El coaching se basa en una conversación especial que le llamamos posibilitadora del cambio entre el cliente o paciente que desea mejorar, y el coach-salud que utiliza habilidades de comunicación para ayudarle a conseguirlo" (Molins, 2011, pp. 6-7).

Respecto a los temas que aborda el coaching de salud, el término suele ser un cajón de sastre que aglutina tanto el coaching de prevención de enfermedades, como el coaching para personas que quieren perder peso, es decir, aquel coaching que les ayuda a alcanzar metas de salud, o el coaching para pacientes en tratamiento o que necesitan un seguimiento. De hecho, Bonal et al. (2012) describen cómo el coaching puede empoderar a los pacientes por medio del diseño conjunto de un plan de acción donde el paciente es protagonista de las tareas (Bonal et al., 2012).

Al utilizar al profesional del coaching como elemento definitorio de este tipo de coaching que trabaja con pacientes, también se registra una falta de acuerdo en lo que respecta a su figura.

Según Bennet et al., (2010), un coach de salud puede ser una persona con o sin formación clínica (enfermera, paciente o trabajador social que han asistido al menos a dos sesiones de formación relacionadas con temas en su mayor parte médicos. Kreisberg (2015) estableció una distinción entre coach de salud (health coach), y coach de bienestar (wellness coach). El primero trabaja con personas con problemas de salud a menudo crónicos, mientras que el segundo se orienta más a la prevención y el bienestar. Construyendo sobre esta definición, Huffman (2016, pp. 490-498) otorga la 
Nuevas necesidades comunicativas del paciente y su relación con el coaching para pacientes

responsabilidad final de la salud del paciente al profesional, que, según las guías de la Sociedad Nacional de Coaches de Salud, tendría que contar con educación y formación clínicas.

Shearsmith (2011, pp. 120-138) sugiere que tanto los coaches de vida, es decir, aquellos con una formación específica de coaching, como los coaches de salud, aquellos con formación clínica y de coaching, podrían llevar a cabo el tipo de coaching al que hacemos referencia en este epígrafe (Caldwell et al., 2013), por su parte, centran la educación de los coaches en una formación de coaching de salud integrativa, sin mencionar la necesidad de formación clínica.

Además del coach de salud, el paciente experto ha encontrado un lugar en el sistema de salud de los últimos años. Su rol puede variar, desde aquel que es capaz de cuidar de sí mismo, hasta el que forma y acompaña a otros pacientes en su camino. A veces los pacientes expertos se convierten en defensores del paciente, teniendo la posibilidad de formarse y mejorar sus habilidades y capacidades (Hunter, et al., 2018, p. 270) para poder involucrarse en todo el proceso de investigación y desarrollo médico (Klingmann et al., 2018 , p. 251). En algunos casos, el rol de los pacientes expertos ha llegado a profesionalizarse, aunque en la gran mayoría se mantienen como voluntarios. La formación que adquiere el paciente experto no se asemeja en ninguno de estos casos a la de un coach profesional.

\subsection{El coaching para pacientes}

En base a las necesidades tanto de comunicación como de relación que manifiesta el paciente de hoy en día y teniendo en cuenta la ambigüedad existente en torno al concepto de coaching de salud, y avalados por los resultados de la investigación llevada a cabo por Ferreiro (2021) con paciente de cáncer de mama, se propone un nuevo tipo de coaching, diseñado específicamente para los pacientes, caracterizado por los siguientes objetivos que en algunos casos ya han sido descritos por otros autores:

- Obtener herramientas que ayuden a los pacientes en su día a día, tanto a gestionar sus emociones, su situación y su vida (Taleghani et al., 2014).

- Dedicarse un espacio seguro y un tiempo propio que favorezca la exploración y reflexión personal (Mok y Martinson, 2000, pp. 2016-213).

- Recuperar la confianza en sí mismos y aprender a ocupar el espacio que merecen (Carpenter et al., 1999, pp. 1402-1411).

- Renovar energías, recuperar la ilusión por el futuro y dejar atrás el miedo a la enfermedad o a lo que pueda ocurrir.

Por otra parte, en base al tipo de profesional responsable de acompañar a los pacientes, se propone incluir como atributo importante para fortalecer la relación entre ambos tanto la formación en coaching profesional como la experiencia como paciente que ha pasado por la misma experiencia. 
Nuevas necesidades comunicativas del paciente y su relación con el coaching para pacientes

Por tanto, el perfil ideal del coach que trabaja con pacientes es un profesional en el que convergen la formación de coach profesional y la condición de paciente, caracterizado por los siguientes aspectos:

- El coach de pacientes no pertenece al equipo médico, sino que al igual que en cualquier otra relación de coaching, se posiciona como un igual ante el resto de pacientes. Por tanto, no se requiere formación médica.

- El coach de pacientes es un coach profesional que ha adquirido el conocimiento y dominio de herramientas y técnicas de coaching y es capaz de realizar intervenciones de coach espontáneas con algunos de los participantes a lo largo de la implementación del modelo.

- El coach comparte con los pacientes un mismo diagnóstico. Haber vivido experiencias similares ayuda a crear confianza y empatía en el grupo porque, como han indicado pacientes que han colaborado en el estudio, "además de sus competencias profesionales, la coach sabe de lo que habla porque ha pasado por lo mismo que nosotras". Conscientes de las limitaciones de este requisito, podrían ser coach es de pacientes aquellos coaches que, si bien no comparten el diagnóstico, lo han vivido muy de cerca (familiar o amigo cercano).

- El coach de pacientes no es responsable de la salud del paciente. Su rol consiste en hacer que el paciente adquiera esa responsabilidad hacia sí mismo.

\section{CONCLUSIONES}

Tanto desde un punto de vista comunicativo como relacional, el posicionamiento del paciente frente a los profesionales de la salud, así como sus necesidades, han evolucionado con el paso de los años. Si bien hasta mediados del siglo XX la relación entre ambos era asimétrica, a partir de ese momento el paciente comienza a ganar poder, adquiriendo más autonomía y capacidad de elección.

El paciente del siglo XXI es un paciente activo, que busca herramientas que le permitan tomar decisiones relacionadas con su enfermedad y con su vida, basándose en su propio criterio. Entre las habilidades que se manifiestan como necesarias en el proceso de comunicación entre el paciente y el equipo médico, destacan la escucha activa, que facilita la apertura de las personas, la empatía, que contribuye a que el paciente se sienta acompañado, las preguntas abiertas, que permiten establecer una relación de confianza basada en un objetivo común, y la mirada atenta, aquella que nace del respeto y contribuye a crear un entorno seguro.

Si bien la disciplina del coaching profesional aúna en su práctica estas habilidades y abre la puerta a un nuevo modelo de relación con el paciente, en aras de su empoderamiento, el análisis de las distintas modalidades del coaching de salud y de sus profesionales deja entrever un vacío que el coaching para pacientes planteado en este artículo y definido a partir de la investigación con pacientes expuesta en la tesis de Ferreiro, se propone ocupar.

El coaching para pacientes es una nueva forma de comunicarse y relacionarse con los pacientes, cuyo objetivo es proporcionarles aquello que necesitan con vistas a su

Revista de Comunicación y Salud, 2021, Vol. 11, 77-104 
Nuevas necesidades comunicativas del paciente y su relación con el coaching para pacientes

empoderamiento, en línea con las demandas del paciente del siglo XXI. Se caracteriza por una serie de elementos que los pacientes describen como clave en esta nueva relación:

- Es un modelo diseñado específicamente para los pacientes y sus necesidades concretas.

- El coach profesional que practica el coaching para pacientes no pertenece al equipo médico.

- El coach comparte diagnóstico con el paciente.

- El paciente adquiere responsabilidad en la relación con su afección.

- El paciente obtiene herramientas que le ayudan en su día a día.

- Los objetivos de la relación se centran en el presente y en el futuro, con vistas a recuperar la ilusión y dejar atrás el miedo a lo que pueda ocurrir.

\section{REFERENCIAS}

Alarcón Orozco, E. (2020). Un proyecto para trabajar la inteligencia espiritual a través de coaching. Revista de Ciencias de la Comunicación e Información, 25(2), 91-105. http://doi.org/10.35742/rcci.2020.25(2).91-105

Ambady, N., LaPlante, D. A., Nguyen, T. B., Rosenthal, R., Chaumeton, N. R. y Levinson, W. S. (2002). Surgeons' tone of voice: a clue to malpractice history. Surgery, 132(1), 5-9. https://doi.org/10.1067/msy.2002.124733

Asnani, M. (2009). Patient-Physician Communication. West Indian Med J, 58(4), 357-361.

Barquero Cabero, M., Rodríguez Terceño, J., Gonzálvez Vallés, J. E. (2018). Tecnologías de la Comunicación y posverdad: implicaciones para la gestión de la Comunicación Hospitalaria. Revista de Comunicación y Salud, 8(1), 85-97. http://doi.org/10.35669/revistadecomunicacionysalud.2018.8(1).85-97

Barrientos-Báez, A. (2019a). El desarrollo de la Inteligencia Emocional en los estudios del Grado en Turismo en la Universidad de La Laguna (Tenerife). Tesis Doctoral. Universidad Camilo José Cela. https://bit.ly/3wWEPnk

Barrientos-Báez, A. (2019b). Redes sociales, salud física e inteligencia emocional. En VV.AA. Tecnología Digital para la Salud y la Actividad Física, 291-315. Pirámide.

Barrientos-Báez, A., Barquero-Cabrero, M. y Rodríguez-Terceño, J. (2019). La educación emocional como contenido transversal para una nueva política educativa: el caso del grado de turismo. Revista Utopía y Praxis Latinoamericana, 24(4), 147-165. https://produccioncientificaluz.org/index.php/utopia/article/view/29796

Belles, B. (2018). Empathic Communication in Healthcare Settings: A patient placebo? EALTHY magazine, 10-11. https://ealthy.com 
Nuevas necesidades comunicativas del paciente y su relación con el coaching para pacientes

Bennet, D. H., Coleman, E. A., Parry, C., Bodenheime, T. y Chen, H. E. (2010). Health coaching for patients with chronic illness. Family Practice Management, 17(5), 24-29. https://www. aafp.org/

Bonal, R., Almenares, H. y Marzan, M. (2012). Coaching de salud: un nuevo enfoque en el empoderamiento del paciente con enfermedades crónicas no transmisibles. Medisan, 16(5).

Borrell, F. (2004). Entrevista Clínica. Manual de estrategias prácticas. Semfyc ediciones. https://www.semfyc.es

Brock, W. G. (2017). Guía de la Historia del coaching. 3ª ed. Uno editorial.

Burt, J., Abel, G., Elliott, M. N., Elmore, N., Newbould, J., Davey, A., Llanwarne, N., Maramba, I., Paddison, C., Campbell, J. y Roland, M. (2018). The Evaluation of Physicians' Communication Skills From Multiple Perspectives. The Annals of Family Medicine, 16 (4), 330-337. https://doi.org/10.1370/afm.2241

Caldwell, K. L., Gray, J. y Wolever, R. Q. (2013). The Process of Patient Empowerment in Integrative Health Coaching: How Does It Happen? Global advances in health and medicine, 2(3), 48-57. https://doi.org/10.7453/gahmj.2013.026

Carpenter, J. S., Brockopp, D. Y. y Andrykowski, M. A. (1999). Self-transformation as a factor in the self-esteem and well-being of breast cancer survivors. Journal Adv. Nurse, 29(6), 1402-11. https://doi.org/10.1046//.1365-2648.1999.01027.x

Carretero-Díaz, M. A. y Barrientos-Báez, A. (2019). Necesidad de formar a los cuidadores de personas en situación de dependencia: comunicación y respuesta desde la UCM. $\begin{array}{lllll}\text { Revista de Comunicación y } & \text { Salud, }\end{array}$ http://doi.org/10.35669/revistadecomunicacionysalud.2019.9(1).19-38

CBC. (2018). How cancer coaches help patients navigate an overwhelming diagnosis. Obtenido de CBC Radio: www.cbc.ca/radio/whitecoat/how-cancer-coaches-helppatients-navigate-an-overwhelming-diagnosis-1.4878003

Clark, M. y Douglas, K. V. (2014). The Effectiveness of Wellness Coaching for Improving Quality of Life. Mayo Clinic Proceedings, 89(11), 1537-1544. https://doi.org/10.1016/j.mayocp.2014.04.028

Cofré Soto, E. (2020).Comunicación para la salud: aproximaciones epidemiológicas y socioculturales al cuerpo enfermo de las mujeres con cáncer de mama. Revista de Comunicación de la SEECI, 51, 169-183. http://doi.org/10.15198/seeci.2020.51.169$\underline{183}$

Compte-Pujol, M., Marca-Francès, G., Menéndez-Signorini, J. y Frigola-Reig, J. (2020). Necesidades de información en pacientes con enfermedades crónicas. ¿Cómo evitar 
Nuevas necesidades comunicativas del paciente y su relación con el coaching para pacientes

el ruido en la relación médico-paciente?. Revista Latina de Comunicación Social, 75, 207-223. www.doi.org/http://doi.org/10.4185/RLCS-2020-1423

Correia, J., Gonçalves, L. y Cabral, A. (2016). The contribution of professional coach for emotional and psychological adjustment of soccer player. Journal of Physical Education, 27, e2703. Epub Julio 14, 2016. https://doi.org/10.4025/JPHYSEDUC.V2711.2703

Cox, E. y Patrick, C. (2012). Managing Emotions at Work: How Coaching Affects Retail Support Workers' Performance and Motivation. International Journal of Evidence Based Coaching and Mentoring, 10(2), 34-51. https://radar.brookes.ac.uk/

Davis, P. A. y Davis, L. (2016). Emotions and emotions regulation in coaching. The psychology of effective coaching and management. Sports and athletics preparation, performance, and psychology, 285-306. Nova Science Publishers.

DiMatteo, M. R. (1998). The Role of the Physician in the Emerging Health Care Environment. Western Journal of Medicine, 168(5), 328-333. www.ncbi.nlm.nih.gov/pmc/articles/PMC1304975/

Emanuel, E. y Emanuel, L. (1999). Cuatro modelos de la relación médico-paciente. Bioética para clínicos, 109-126.

Esquirol, J. M. (2006). El respeto o la mirada atenta. Editorial Gedisa.

Esquirol, J. M. (2021). Filosofía como mirada atenta. Revista internacional de filosofía aplicada HASER, 125-144. https://revistascientificas.us.es/index.php/HASER/article/view/15073

Ferguson, T. y e-Patients Scholars Working Group. (2007). E-patients. How they can help us heal health care. Robert Wood Johnson Foundation Quality Health Care .

Ferreiro, T. (2021). La comunicación y el coaching como instrumentos de empoderamiento del paciente [manuscrito no publicado]. Madrid: Comunicacion audivisual III. Ciencias de la Información. Universidad Complutense.

Fong Ha, J. y Longnecker, N. (2010). Doctor-Patient Communication: A Review. The Ochsner Journal, 10(1), 38-43.

García-Manso, Angélica (2021). Iconografías en prensa digital sobre la pandemia de COVID-19: un retrato cultural del coronavirus en España en abril de 2020. Vivat Academia, Revista de Comunicación, 154, 45-61. https://doi.org/10.15178/va.2021.154.e1234

Gifford, K. A. y Fall, L. H. (2014). Doctor Coach. Academic Medicine, 89(2), 272-276. https://doi.org/10.1097/ACM.0000000000000097 
Nuevas necesidades comunicativas del paciente y su relación con el coaching para pacientes

Goleman, D. (1996). Inteligencia Emocional. Kairós.

Gray, R. E., Doan, B. D. y Church, K. (1991). Empowerment and Persons with Cancer: Politics in Cancer Medicine. Journal of Palliative Care, 6(2), 33-45. https://doi.org/10.1177/082585979000600206

Hellín, T. (2002). The Physician-patient relationship: recent developments and changes. Haemophilia, 8, 450-454.

Honavar, S. G. (2018). Patient-physician relationship-Communication is the key. Indian journal of ophthalmology, 66(11), 1527-1528. https://doi.org/10.4103/ijo.IJO 176018

Huffman, M. (2009). Health Coaching: A Fresh, New Approach to Improve Quality Outcomes and Compliance for Patients with Chronic Conditions. Home Healthcare Nurse: The Journal for the Home Care and Hospice Professional, 27(8), 490-498. https://doi.org/10.1097/01.NHH.0000360924.64474.04

Hunter, A., Facey, K., Thomas, V., Haerry, D., Warner, K., Klingmann, May, M. y See, W. (2018). EEUPATI Guidance for Patient Involvement in Medicines Research and Development: Health Technology Assessment. Frontiers in Medicine, 5, 270. https://doi.org/10.3389/fmed.2018.00270

ICF. (2018, 1 julio). ICF., Definición de coaching: www.icfes.com/mwsicf/sobreicf/definicion-coaching-icf-espana

Kabaa, R. y Sooriakumaran, P. (2007). The evolution of the doctor-patient relationship. International Journal of Surgery, 5(1), 57-65. https://doi.org/10.1016/j.ijsu.2006.01.005

Karora, N. (2003). Interacting with cancer patients: the significance of physicians' communication behavior. Social Science \& Medicine, 57(5), 791-806. https://doi.org/10.1016/S0277-9536(02)00449-5

Kimsey-House, H., Kimsey-House, K., Sandahl, P. y Whitworth, L. (2011). Co-active coaching. Changing businesses, transforming lives. (3rd edition). Nicholas Brealey Publishing.

Klingmann, I., Heckenberg, A., Warner Kay, H. D., Hunter, A., Matthew, M. y Wolf, S. (2018). EUPATI and Patients in Medicines Research and Development: Guidance for Patient Involvement in Ethical Review of Clinical Trials. Frontiers in Medicine, 251. https://doi.org/10.3389/fmed.2018.00251

Knapp, M. L. (1980). La comunicación no verbal - El cuerpo y el entorno. Paidós Comunicación. 
Nuevas necesidades comunicativas del paciente y su relación con el coaching para pacientes

Kreisberg, J. (2015, 8 de septiembre). Health coaching in a clinical setting. Teleosis Institute: https://teleosis.org/health-coaching-in-a-clinical-setting/

Lara Martínez, M. y Lara Martínez, A. (2018). Prejuicios y estereotipos en el cine sobre trastornos alimentarios. Revista de Comunicación y Salud, 8(2), 21-39. http://doi.org/10.35669/revistadecomunicacionysalud.2018.8(2).21-39

Lázaro, J. y Gracia, D. (2006). La relación médico-enfermo a través de la historia. Anales del Sistema Sanitario de Navarra, 29(3), 7-17. https://bit.ly/30qlpb6

Leonard, T. J. (2001). Coachville. 100 Coaching Principles: www.coachville.com/prin/coachingprinciplespdf.pdf

Lichstein, P. R. (1990). The Medical Interview. En H. W. Walker HK, Clinical Methods: The History, Physical, and Laboratory Examinations. 3rd edition. (pp. 29-36. www.ncbi.nlm.nih.gov/books/NBK201/.

López del Castillo Wilderbeek, F. L. (2021). El seguimiento sobre las fake news en medios institucionales durante el coronavirus en España. Vivat Academia, Revista de Comunicación, 154, 1-12. https://doi.org/10.15178/va.2021.154.e1253

López Meneses, E., Vázquez Cano, E., Fernández Márquez, E. y López Belmonte, J. (2019). Representaciones conceptuales multimedia de los ámbitos de actuación en educación social elaborados por estudiantes universitarios (2015-2018). Revista de Comunicación de la SEECI, 50, 27-42. https://doi.org/10.15198/seeci.2019.50.27-42

Lucas Moreno, M. L. (2020). Importancia y sentido del coaching y la inteligencia emocional en itinerarios para el empleo de personas con discapacidad intelectual. Revista de Ciencias de la Comunicación e Información, 25(2), 41-53. http://doi.org/10.35742/rcci.2020.25(2).41-53

Maguire, P. (1999). Improving communication with cancer patients. European Journal of Cancer, 35(10), 1415-1422. https://doi.org/10.1016/s0959-8049(99)00178-1

McCabe, R. y Healey, P. G. (2018). Miscommunication in Doctor-Patient Communication. Topics in Cognitive Science, 10(2), 409-424. https://doi.org/10.1111/tops.12337

Mead, N. y Bower, P. (2000). Patient-centredness: a conceptual framework and review of the empirical literature. Social Science \& Medicine, 51(7), 1087-1110. https://doi.org/10.1016/s0277-9536(00)00098-8

Mestre, A. G. (2015). Programa Pacient Expert Catalunya®: una estratègia per potenciar l'autorresponsabilitat del pacient i el foment de l'autocura. Barcelona: Generalitat de Catalunya. Programa de Prevenció i atenció a la cronicitat de Catalunya. 
Nuevas necesidades comunicativas del paciente y su relación con el coaching para pacientes

Mok, E. y Martinson, I. (2000). Empowerment of Chinese Patients With Cancer Through Self-Help Groups in Hong Kong. Cancer Nursing, 23(3), 206-213. https://doi.org/10.1097/00002820-200006000-00008

Molins, J. (2011). Coaching y Salud. Cuadernos de Coaching, 6-7.

Muñoz, M., Ruiz, R. y Pérula, L. A. (2002). Evaluation of a patient-centred approach in generalized musculoskeletal chronic pain/fibromyalgia patients in primary care. Patient Education and Counseling, 48(1), 23-31. https://doi.org/10.1016/s07383991(02)00095-2

Odor Hurtado, E. J., Portugal de la Cuerda, P., Salado García, P. y Sanz Gómez, O. (2020). Programa para el fortalecimiento de la autoestima en jugadoras de fútbol adolescentes. Revista de Ciencias de la Comunicación e Información, 25(2), 157-182. http://doi.org/10.35742/rcci.2020.25(2).157-182

Pellegrini C. A. (2017). Trust: The Keystone of the Patient-Physician Relationship. Journal of the American College of Surgeons, 224(2), 95-102. https://doi.org/10.1016/j.jamcollsurg.2016.10.032

Pinto, R. Z., Ferreira, M. L., Oliveira, V. C., Franco, M. R., Adams, R., Ferreira, P. H. y Zambelli Pinto, R. (2012). Patient-centred communication is associated with positive therapeutic alliance: a systematic review. Journal of Physiotherapy, 58(2), 77-87. https://doi.org/10.1016/S1836-9553(12)70087-5

Piqueiras Conlledo, P., De Marchis, G. y Cuesta Díaz, V. (2020). Análisis del contenido publicado en YouTube, Facebook e Internet sobre vacunas y anti-vacunas. Revista de Comunicación y Salud, 10(1), 67-90. http://doi.org/10.35669/rcys.2020.10(1)

Platón (1988). Las leyes. Ediciones Akal.

Programa Pacient expert Catalunya. (2013 30 de septiembre). Canal Salut - Generalitat de Catalunya: https://bit.ly/30glw6w

Rando Cueto, D., De las Heras Pedrosa, C. y Paniagua Rojano, F. J. (2021). Influencia de las redes sociales en el trabajo de profesionales de la información especializados en salud. Caso de los Colegios Oficiales de Médicos de España. Revista Latina de Comunicación Social, 79, 113-133. https://doi.org/10.4185/RLCS-2021-1491

Roberts, K. (1999). Patient empowerment in the United States: a critical commentary. Health Expect., 2(2), 82-92. https://doi.org/10.1046/j.1369-6513.1999.00048.x

Rosenberg, M. y Chopra, D. (2015). Nonviolent Communication: A Language of Life, 3rd Edition: Life-Changing. 
Nuevas necesidades comunicativas del paciente y su relación con el coaching para pacientes

Rosenberg, M. y Molho, P. (1998). Nonviolent (empathic) communication for health care providers. Haemophilia: the official journal of the World Federation of Hemophilia, 4(4), 335-340. https://doi.org/10.1046/.1365-2516.1998.440335.x

Sancho Escrivá, J. V., Fanjul Peyró, C., De la Iglesia Vayá, M., Montell, J. A. y Escartí Fabra, M. J. (2020). Aplicación de la inteligencia artificial con procesamiento del lenguaje natural para textos de investigación cualitativa en la relación médico-paciente con enfermedad mental mediante el uso de tecnologías móviles. Revista de Comunicación y Salud, 10(1), 19-41. http://doi.org/10.35669/rcys.2020.10(1).19-41

Servellen, G. V. (2009). Communication Skills for the Health Care Professional. Jones and Bartlett Publishers.

Shearsmith, A. (2011). An exploration of holistic life coaching for breast cancer survivors. International Journal of Evidence Based Coaching and Mentoring, 5, 120-138. https://bit.ly/30m9hYT

Simón, P. (2006). Diez mitos en torno al consentimiento informado. Anales del Sistema Sanitario de Navarra, 29(3), 29-40. http://dx.doi.org/10.4321/S1137$\underline{66272006000600004}$

Szasz, T. y Hollender, M. (1956). A contribution to the philosophy of medicine: the basic model of the doctor-patient relationship, 97, 585-592. Arch Int Med.

Taleghani, F., Bahrami, M., Loripoor, M. y Yousefi, A. (2014). Empowerment Needs of Women With Breast Cancer: A Qualitative Study. Iranian Red Crescent medical journal, 16(11), e16379. https://doi.org/10.5812/ircmj.16379

Timmermans, S. (2020). The Engaged Patient: The Relevance of Patient-Physician Communication for Twenty-First-Century Health. Journal of Health and Social Behavior. 16(3), 259-273. https://doi.org/10.1177/0022146520943514

TV3, (2019, 8 Marzo). Noticies vespre. Metges que han estat pacients creen el grup Janus per fer més humà el sistema sanitari. https://bit.ly/3rr9PJ1 [Película]

Vargas Delgado, J. J. (2020). Stress coaching 0.0. Experimental programa de coaching para reducir el estrés. Revista de Ciencias de la Comunicación e Información, 25(2), 71-90. http://doi.org/10.35742/rcci.2020.25(2).71-90

Veliz, A. L., Dörner, A. P., Salcedo, A. S., Reyes, J. L. y Ganga-Contreras, F. (2018). Inteligencia emocional y bienestar psicológico en profesionales de enfermería del sur de Chile. Revista Medisur, 16(2).

Whitmore, J. (2010). Coaching for Performance: GROWing Human Potential and Purpose - the Principles and Practice of Coaching and Leadership. Quercus. 
Nuevas necesidades comunicativas del paciente y su relación con el coaching para pacientes

World Health Organization (2009). WHO Guidelines on Hand Hygiene in Health Care: First Global Patient Safety Challenge Clean Care Is Safer Care. Geneva. https://www.ncbi.nlm.nih.gov/books/NBK144004/

World Health Organization (1998). Health Promotion Glossary. Geneva: World Health Organization.

Yeoman, G., Furlong, P., Seres, M., Binder, H., Chung, H., Garzya, V. y Jones, R. (2017). Defining patient centricity with patients for patients and caregivers: a collaborative endeavour. BMJ Innovations 3, 76-83.

\section{AUTORES}

\section{Teresa Ferreiro Vilariño}

Licenciada en Ciencias de la Información por la Universidad Complutense de Madrid. Teresa compagina el trabajo en su tesis en esta misma facultad (fecha de publicación prevista en junio 2021) con su actividad profesional como coach profesional certificada por ICF. Con más de diez años de experiencia trabajando con pacientes de cáncer de mama desde el momento de su diagnóstico como afectada, Autora del libro Un bulto en la mama, tú eliges cómo abordarlo (2012) y ponente asidua en eventos dirigidos tanto a pacientes como a profesionales del sector salud, en torno al tema del empoderamiento del paciente. En 2019, EyeforPharma reconoció su trayectoria y su labor al otorgarle el European Patient Champion Award. Teresa es además la fundadora de Baby Beatles, una organización sin ánimo de lucro que ayuda a mujeres que han tenido cáncer de mama a convertirse en madres, y trabaja actualmente en Soul Reconnect, un innovador proyecto de empoderamiento del paciente a través del coaching para pacientes, término que ha acuñado a partir de la investigación realizada en su tesis doctoral. Coach de liderazgo en la prestigiosa escuela de negocios suiza IMD.

Orcid ID: https://orcid.org/0000-0002-6687-7820

Google Scholar: https://scholar.google.com/citations?hl=en\&user=Ved2QM0AAAAJ

ResearchGate: https://www.researchgate.net/profile/Teresa-Ferreiro 